\title{
RESEARCH
}

\section{Development of a triage protocol for critical care during an influenza pandemic}

\author{
Michael D. Christian, Laura Hawryluck, Randy S. Wax, Tim Cook, Neil M. Lazar, Margaret S. Herridge, \\ Matthew P. Muller, Douglas R. Gowans, Wendy Fortier, Frederick M. Burkle, Jr.
}

$\infty$

See related article page $\mathrm{I} 393$

\section{ABSTRACT}

Background: The recent outbreaks of avian influenza $\left(\mathrm{H}_{5} \mathrm{~N}_{1}\right)$ have placed a renewed emphasis on preparing for an influenza pandemic in humans. Of particular concern in this planning is the allocation of resources, such as ventilators and antiviral medications, which will likely become scarce during a pandemic.

Methods: We applied a collaborative process using best evidence, expert panels, stakeholder consultations and ethical principles to develop a triage protocol for prioritizing access to critical care resources, including mechanical ventilation, during a pandemic.

Results: The triage protocol uses the Sequential Organ Failure Assessment score and has 4 main components: inclusion criteria, exclusion criteria, minimum qualifications for survival and a prioritization tool.

Interpretation: This protocol is intended to provide guidance for making triage decisions during the initial days to weeks of an influenza pandemic if the critical care system becomes overwhelmed. Although we designed this protocol for use during an influenza pandemic, the triage protocol would apply to patients both with and without influenza, since all patients must share a single pool of critical care resources.

CMAJ 2006;175(II):I377-8I

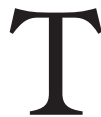
he recent outbreaks of avian influenza $\left(\mathrm{H}_{5} \mathrm{NI}^{1-4}\right.$ have placed a renewed emphasis on preparing for an influenza pandemic in humans. ${ }^{5,6}$ Developing plans to deal with the allocation of critical care resources, including mechanical ventilators, which will likely become scarce during a pandemic, ${ }^{6-8}$ presents a significant challenge. Health care systems in the developed world have rarely encountered the type of resource scarcities envisaged during an influenza pandemic. Models of the potential impact of a pandemic on the Ontario population predict that hospital admissions for influenza will peak at 1823 per day over a 6 week period. ${ }^{9}$ This translates to $72 \%$ of the total hospital capacity being used by influenza patients. Similarly, the de- mand for intensive care unit (ICU) resources, solely for patients with influenza, would peak at $17 \mathrm{I} \%$ of current ICU bed capacity and II $8 \%$ of the ventilator capacity. These figures do not take into account the current usage rate of critical care for patients without influenza, which is nearly at I00\%. Nor does this model factor in the availability of human resources. Surge response strategies ${ }^{10}$ (e.g., scaling back elective procedures, opening additional critical care areas and implementing the use of "mass critical care"11,12) will partially mitigate the sudden demand for medical care during an influenza pandemic; however, these strategies will be inadequate to fully address the demands on the health care system.

When resource scarcities occur, the tenets of biomedical ethics and international law dictate that triage protocols be used to guide resource allocation. ${ }^{13-15}$ International law requires a triage plan that will equitably provide every person the "opportunity" to survive. However, such a law does not guarantee either treatment or survival. ${ }^{16}$ We have developed this triage protocol in an effort to ensure the equitable and efficient use of critical care resources if scarcities occur during an influenza pandemic.

\section{Methods}

In December 2004, at the request of the steering committee of the Ontario Health Plan for an Influenza Pandemic $(\mathrm{OH}-$ PIP), a group of clinicians with expertise in critical care, infectious diseases, medical ethics, military medicine, triage and disaster management was convened to provide advice regarding critical care during an influenza pandemic. They specifically sought to address issues surrounding critical care resource allocation.

The working group undertook an in-depth review of the medical literature by searching MEDLINE for articles published from January Ig66 to December 2004 using the terms "triage," "intensive care," "critical care," "mechanical ventilation," "influenza" and "pandemic." The group also conducted an in-depth review of the disaster literature, military protocols and published pandemic plans as well as Internet searches and networking with experts in critical care medicine, military medicine and disaster medicine to identify any 
existing critical care triage protocols or to find information necessary to develop a protocol if none existed. Preliminary results of this work, including a prototype triage protocol, were incorporated into the June 2005 version of the OHPIP. ${ }^{7}$ The prototype protocol was then refined in January 2006 on the basis of input from leading experts in a wide variety of relevant medical disciplines across Ontario.

Subsequently I5O critical care leaders in the province were invited to comment on the final report of the working group, which included the triage protocol. Fifty-five of these leaders reviewed the report and provided feedback, which was used to improve the clarity of the protocol. In this article we present our proposed protocol for critical care triage during an influenza pandemic.

\section{Results}

The literature search, Internet searches and consultations with experts failed to identify any general triage protocols for critical care. Therefore, the working group embarked on developing a critical care triage protocol. Although triage protocols were identified for trauma $\mathrm{a}^{17-20}$ and chemical, biological, radiation and nuclear events, ${ }^{16}$ these were not felt to be generalizable to the population or situations in critical care. However, certain features of these protocols (e.g., a colour-coded triage tool, ${ }^{21}$ inclusion criteria, exclusion criteria and minimum qualifications for survival ${ }^{16}$ ) were deemed useful for a triage protocol for critical care. Illness severity scoring systems used to predict critical care outcomes were also assessed for potential inclusion in the protocol. ${ }^{22-27}$ The Sequential Organ Failure Assessment (SOFA) score was felt to be the most appropriate scoring system for use in a triage protocol given its basis on physiologic parameters, ease of calculation, requirement for simple laboratory tests and its validation for use in patients with a wide variety of conditions requiring critical care..$^{22,28-31}$ (The scoring criteria for the SOFA score are provided in Ap- pendix I, available online at www.cmaj.ca/content/cgi/full/I75 /II/I377/DCr.) The final protocol developed by the working group is presented in part in Fig. I and in Box I and Box 2.

The protocol is designed to provide guidance for making triage decisions during the initial days to weeks of an influenza pandemic if the critical care system is overwhelmed. The impetus for this triage protocol was the potential for the critical care system to be flooded by patients with influenza during a pandemic. However, it must be noted that the triage protocol would apply to all patients who are being considered for critical care, not just those with influenza, since all patients must share a single pool of resources.

The triage protocol has 4 main components: inclusion criteria, exclusion criteria, minimum qualifications for survival and a prioritization tool. The inclusion criteria (Box 2) identify patients who may benefit from admission to critical care and primarily focus on respiratory failure, since the provision of ventilatory support is what fundamentally differentiates the ICU from other acute care areas (e.g., step-down units). It is anticipated that expanded care models will be developed as part of the surge capacity response and will permit hemodynamic support and other advanced care modalities to be provided in areas that have appropriate monitoring but do not typically provide this level of care. However, should hemodynamic support not be available elsewhere, it will qualify as an inclusion criterion for critical care admission.

The exclusion criteria (Box 2) can be broken down into 3 categories: patients who have a poor prognosis despite care in an ICU, patients who require resources that simply cannot be provided during a pandemic and patients with advanced medical illnesses whose underlying illness has a poor prognosis with a high likelihood of death, even without their current concomitant critical illness. The first category reflects the "hard" boundaries that many intensivists recognize from their day-to-day care of patients, ${ }^{32-35}$ such as end-stage cancer, severe burns,${ }^{36}$ severe trauma, ${ }^{37,38}$ unwitnessed or recur-

\begin{tabular}{|c|c|c|}
\hline Triage code & Criteria & Action or priority \\
\hline Blue & Exclusion criteria met or SOFA score $>11^{*}$ & $\begin{array}{l}\text { - Manage medically } \\
\text { - Provide palliative care as needed } \\
\text { - Discharge from critical care }\end{array}$ \\
\hline Red & SOFA score $\leq 7$ or single-organ failure & Highest priority \\
\hline Yellow & SOFA score $8-11$ & Intermediate priority \\
\hline Green & No significant organ failure & $\begin{array}{l}\text { - Defer or discharge } \\
\text { - Reassess as needed }\end{array}$ \\
\hline
\end{tabular}

Note: SOFA = Sequential Organ-Failure Assessment.

*If an exclusion criterion is met or the SOFA score is > 11 anytime from the initial assessment to 48 hours afterward, change the triage code to Blue and proceed as indicated.

Fig 1: Prioritization tool used in triage protocol for the initial assessment of patients' needs for critical care during an influenza pandemic. See online Appendix 1 for the SOFA scoring criteria and online Appendix 2 for the complete prioritization tool, which includes details on reassessing patients at 48 and 120 hours (appendices are available at www.cmaj.ca/cgi/content/full/175/11/1377/DC1). See Box 2 for exclusion criteria. 
rent cardiac arrests ${ }^{39}$ and patients with a baseline SOFA score greater than II, who have more than a $90 \%$ mortality. ${ }^{22,28} \mathrm{Ad}-$ vanced age clearly contributes to higher death rates in specific disease subsets of critically ill patients ${ }^{40,41}$ but it may not be a strong predictor of critical care outcomes in general and therefore was not included in the original draft protocol. However, we received strong and consistent feedback from both expert and stakeholder consultations that an age criterion should be included. This remains one of the areas requiring further research and community input.

The second category of exclusion criteria includes patients who may benefit from critical care but would require intense use of resources and prolonged care that cannot be justified during a pandemic, when the goal is to do the most for the most with the limited resources available. The third category of exclusion criteria includes patients who have high resource requirements and are likely to experience significant complications from influenza (e.g., patients with advanced cancer or immunosuppression). Others in this category are patients who have end-stage cardiac, hepatic or pulmonary failure. The cut-off marks selected here are adapted from the transplant literature ${ }^{42-45}$ and typically represent a baseline death rate higher than $50 \%$ within the next I to 2 years. Given that transplantation is unlikely during a pandemic, combined with the cumulative risk of death from their acute critical illness, these patients again fall into a category where considerable resources would have to be expended with a low probability of long-term survival. Many of the criteria in this third category require more detailed definitions in order to put it into practice. These were omitted for brevity but will be included in the training provided for triage officers.

The "minimum qualifications for survival" form the third component of the triage protocol. These qualifications represent a ceiling on the amount of resources that can be expended on any one person. This is a concept foreign to many medical systems in developed countries but one that has been used in war zones and refugee camps. ${ }^{16}$ The minimum qualifications for survival dictate reassessment at 48 and $\mathrm{I} 2 \mathrm{o}$ hours, as well as an ongoing cut-off ceiling if a patient ever has a SOFA score of

Box 1: Instructions for the application of the triage protocol to determine a patient's need for critical care during an influenza pandemic*

1. Assess whether the patient meets the inclusion criteria

- If yes, proceed to step 2

- If no, reassess patient later to determine whether clinical status has deteriorated

2. Assess whether the patient meets the exclusion criteria $\dagger$

- If no, proceed to step 3

- If yes, assign a "blue" triage code; do not transfer the patient to critical care; continue current level of care or provide palliative care as needed

3. Proceed to triage tool, initial assessment (see Fig. 1)

*The triage protocol applies to all patients undergoing assessment for possible critical care and not only those with influenza-like symptoms. tSee Box 2 for inclusion and exclusion criteria.
Box 2: Detailed inclusion and exclusion criteria used in the triage protocol for critical care during an influenza pandemic

\section{Inclusion criteria}

The patient must have 1 of the following:

A. Requirement for invasive ventilatory support

- Refractory hypoxemia $\left(\mathrm{SpO}_{2}<90 \%\right.$ on non-rebreather mask or $\mathrm{FlO}_{2}>0.85$ )

- Respiratory acidosis $(\mathrm{pH}<7.2)$

- Clinical evidence of impending respiratory failure

- Inability to protect or maintain airway

B. Hypotension (systolic blood pressure $<90 \mathrm{~mm} \mathrm{Hg}$ or relative hypotension) with clinical evidence of shock (altered level of consciousness, decreased urine output or other evidence of end-organ failure) refractory to volume resuscitation requiring vasopressor or inotrope support that cannot be managed in ward setting

\section{Exclusion critiera}

The patient is excluded from admission or transfer to critical care if any of the following is present:

A. Severe trauma

B. Severe burns of patient with any 2 of the following:

- Age $>60$ yr

- > $40 \%$ of total body surface area affected

- Inhalation injury

C. Cardiac arrest

- Unwitnessed cardiac arrest

- Witnessed cardiac arrest, not responsive to electrical therapy (defibrillation or pacing)

- Recurrent cardiac arrest

D. Severe baseline cognitive impairment

E. Advanced untreatable neuromuscular disease

F. Metastatic malignant disease

G. Advanced and irreversible immunocompromise

$\mathrm{H}$. Severe and irreversible neurologic event or condition

I. End-stage organ failure meeting the following criteria: Heart

- NYHA class III or IV heart failure

Lungs

- COPD with $\mathrm{FEV}_{1}<25 \%$ predicted, baseline $\mathrm{PaO}_{2}<55 \mathrm{~mm} \mathrm{Hg}$, or secondary pulmonary hypertension

- Cystic fibrosis with postbronchodilator $\mathrm{FEV}_{1}<30 \%$ or baseline $\mathrm{PaO}_{2}<55 \mathrm{~mm} \mathrm{Hg}$

- Pulmonary fibrosis with VC or TLC $<60 \%$ predicted, baseline $\mathrm{PaO}_{2}<55 \mathrm{~mm} \mathrm{Hg}$, or secondary pulmonary hypertension

- Primary pulmonary hypertension with NYHA class III or IV heart failure, right atrial pressure $>10 \mathrm{~mm} \mathrm{Hg}$, or mean pulmonary arterial pressure $>50 \mathrm{~mm} \mathrm{Hg}$

Liver

- Child-Pugh score $\geq 7$

J. Age $>85 \mathrm{yr}$

$\mathrm{K}$. Elective palliative surgery

Note: $\mathrm{SpO}_{2}=$ oxygen saturation measured by pulse oximetry, $\mathrm{FIO}_{2}=$ fraction of inspired oxygen, NYHA = New York Heart Association, COPD = chronic obstructive pulmonary disease, $\mathrm{FEV}_{1}=$ forced expiratory volume in

1 second, $\mathrm{PaO}_{2}=$ partial pressure of arterial oxygen, $\mathrm{VC}=$ vital capacity, TLC $=$ total lung capacity. 
II or higher or any other exclusion criteria. The key component of the minimum qualifications for survival is the attempt to identify at an early stage patients who are not improving and who are likely to have a poor outcome. In day-to-day practice, it may take days or weeks of intensive care before this poor outcome occurs. During a pandemic, several other patients could have had their lives saved during this time.

The final component of the triage protocol is a tool for the prioritization of patients for admission to the ICU and access to ventilation (Fig. I; see the complete prioritization tool in Appendix 2, available online at www.cmaj.ca/cgi/content /full/I75/II/I377/DCI). For ease of use, the familiar colour scheme (blue or black, red, yellow, green) commonly used in civilian and military disaster triage protocols was adopted. Patients in the blue (or black) category are those who fall into the expectant category and should not receive critical care. Depending on their condition and medical issues, patients may either continue to have curative medical care on a ward or palliative care. Patients in the red category have the highest priority for ICU admission and mechanical ventilation, if required. In selecting the patients for this category, the aim is to find those who are sick enough to require the resource and whose outcome will be poor if they do not receive it but are not so sick that they will not recover even if they do receive ICU care. Patients with single organ failure, particularly those with respiratory failure due to influenza who otherwise have a low SOFA score, are included in the red category assuming they have no exclusion criteria. These patients will derive the maximum benefit from ICU care and mechanical ventilation. The goal is to optimize the effectiveness of the triage protocol so that every patient who receives resources will survive. Although this is unlikely to be completely successful, it can be used as a target to guide modification of the triage protocol based on patient outcomes during a pandemic. Patients in the yellow category are those who, at baseline, are very sick and may or may not benefit from critical care. They should receive care if the resources are available but not at the expense of denying care to someone in the red category. At the reassessment points, patients who are improving are given high priority (red) for continued care, while those who are not showing signs of improvement are classified as yellow. Patients in the green category are those who should be considered for transfer out of the ICU because they are well enough to be cared for without mechanical ventilation or other ICUspecific interventions.

\section{Interpretation}

This triage protocol is a tool aimed at maximizing benefits for the largest number of patients presenting to an overwhelmed critical care system. The ethical values that inform this protocol were derived from the work of the Joint Centre for Bioethics, in the document Stand on Guard for Thee. ${ }^{46}$ The authors identify ro substantive values (individual liberty, protection of the public from harm, proportionality, privacy, duty to provide care, reciprocity, equity, trust, solidarity and stewardship) and 5 procedural values (reasonable, open and transparent, inclusive, responsive and accountable).
Under normal circumstances, all patients should have an equal claim to receive the health care they need. Unfortunately, during a pandemic it will not be possible for all patients to receive intensive care due to finite resources. A triage protocol will assist in distributing the available resources fairly by triaging patients who will not benefit from treatment to noncritical care management, thereby conserving critical care resources for patients who are more likely to benefit. Although it may be unfortunate that some patients do not receive all that they could possibly "use," this does not by default make it unfair. Any restrictions placed on treatment must, however, adhere to the value of proportionality, which requires that restrictions to individual liberties not exceed what is necessary to address the essential needs of the community.

The protocol was developed by experts in advance of a pandemic, to allow time for thoughtful contemplation and community input before ever employing the protocol. When the protocol is implemented, it must be used in an open and transparent manner. Its limitations must be acknowledged, with plans to revise it as information becomes available regarding prognostic factors, specifics of the influenza virus causing the pandemic and resource availability. Processes for ongoing re-evaluation and for consideration of exceptions must be developed in addition to an appeals mechanism. Aside from being an ethical imperative, a process to evaluate and refine the triage protocol prevents undertriage or overtriage, both of which increase overall deaths in disasters. ${ }^{47}$ This process will require a central triage committee as well as appropriate infrastructure to collect and review the information for analysis and to disseminate revisions to the protocol. This committee may serve many different and distant critical care centres. The central triage committee must assure a population-based approach with equitable resource allocation. Advanced training will be required for triage officers to effectively implement the triage protocol. This is not unique to our protocol, since most military and disaster triage guidelines also require extensive training for effective use.

The primary limitation of the current protocol is that it has never been used. Future efforts to test and refine the protocol through modeling and disaster exercises are underway. Such activities will offer the first insight into the true utility of our protocol. A recently published conceptual framework for developing triage guidelines for use in a pandemic includes exclusion criteria similar to those developed by our working group. ${ }^{48}$

This triage protocol for critical care during an influenza pandemic was developed through a collaborative process using best evidence and applying ethical principles. It has been designed to be practical, user-friendly and flexible, but it requires testing in advance of a pandemic. It must be modified as information accrues during a pandemic. A significant lesson learned during this exercise is that development of a triage protocol is a complex process that cannot be undertaken during a pandemic. Similarly, the resource infrastructure required to support effective triage management must also be planned, established and tested before a pandemic. Whether jurisdictions adopt this triage protocol or work to develop alternatives, the time to begin the process is now. 
This article has been peer reviewed.

From the Division of Infectious Diseases and Critical Care, Department of Postgraduate Medicine, McMaster University, Hamilton, Ont. (Christian); the Division of Critical Care, Department of Medicine, University of Toronto (Hawryluck, Wax, Herridge), and University Health Network (Hawryluck, Lazar, Herridge), Toronto, Ont.; the Program for Resuscitation Education and Patient Safety, Department of Medicine, Mount Sinai Hospital, Toronto, Ont. (Wax); Medcan Travel Clinic, Medcan Health Management Inc., Toronto, Ont. (Cook); the Faculty of Medicine and Joint Center for Bioethics (Lazar), the Division of Infectious Diseases, Department of Medicine, and the Department of Health Policy, Management and Evaluation (Muller), University of Toronto, Toronto, Ont.; the Divisions of Acute Services and Community Health, Ontario Ministry of Health and Long-Term Care, Toronto, Ont. (Gowans); the Ottawa Hospital, Ottawa, Ont. (Fortier); and the Asia-Pacific Center for Biosecurity, Disaster and Conflict Research, John A. Burns School of Medicine, University of Hawaii, Honolulu, Hawaii (Burkle)

Competing interests: None declared.

Contributors: All of the authors contributed to the design of the triage protocol and provided intellectual content to the article. All of the authors approved the final version for publication.

Acknowledgement: The Emergency Management Unit of the Ontario Ministry of Health and Long-Term Care provided financial and logistical support for an expert panel retreat to refine the prototype and Internet resources for the stakeholder consultation. The Ontario Ministry of Health and Long-Term Care provided staff support for the working group.

\section{REFERENCES}

I. World Health Organization Global Influenza Program Surveillance Network. Evo-

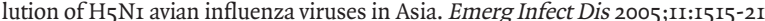

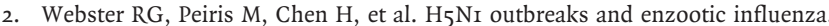
Emerg Infect Dis 2006;12:3-8.

3. Beigel JH, Farrar J, Han AM, et al. Avian influenza A ( $\mathrm{H}_{5} \mathrm{NI}_{\mathrm{I}}$ ) infection in humans. NEngl J Med 2005;353:1374-85.

4. Hayden F, Croisier A. Transmission of avian influenza viruses to and between humans. JInfect Dis 2005;192:1311-4.

5. Fauci AS. Pandemic influenza threat and preparedness. Emerg Infect Dis 2006;:12:73-7.

6. Osterholm MT. Preparing for the next pandemic. NEngl J Med 2005;352:1839-42.

7. Ministry of Health and Long-Term Care. Ontario health plan for an influenza pandemic 2006. Available: www.health.gov.on.ca/english/providers/program/emu Ipan_flu/pan_flu_plan.html (accessed 2006 Sept 25).

8. Public Health Agency of Canada. Canadian pandemic influenza plan. 2004. Available: www.phac-aspc.gc.ca/cpip-pclcpi/ (accessed 2006 Sept 25).

9. US Centers for Disease Control and Prevention (CDC). FluSurge 2.o. Atlanta: CDC Available: www.cdc.gov/flu/flusurge.htm (accessed 2006 Oct I6).

Io. Hick JL, Hanfling D, Burstein JL, et al. Health care facility and community strategies for patient care surge capacity. Ann Emerg Med 2004;44:253-6r.

II. Rubinson L, Nuzzo JB, Talmor DS, et al. Augmentation of hospital critical care capacity after bioterrorist attacks or epidemics: recommendations of the Working Group on Emergency Mass Critical Care. Crit Care Med 2005;33:2393-403.

I2. Rubinson L, O'Toole T. Critical care during epidemics. Critical Care 2005;9:3II-3.

I3. Domres B, Koch M, Manger A, et al. Ethics and triage. Prehospital Disaster Med 200I; 6:53-8.

I4. Baskett PJ. Ethics in disaster medicine. Prehospital Disaster Med i994;9:4-5.

15. Vollmar L.C. Military medicine in war: the Geneva Conventions today. In: Beam TE, Sparacino LR, editors. Military medical ethics. Washington, DC: Office of the Surgeon General;2003.

I6. Burkle FM Jr. Mass casualty management of a large-scale bioterrorist event: an epidemiological approach that shapes triage decisions. Emerg Med Clin North Am 2002;20:409-36.

17. Benson M, Koenig KL, Schultz CH. Disaster triage: START, then SAVE-a new method of dynamic triage for victims of a catastrophic earthquake. Prehospital Disaster Med I996;11:117-24.

I8. Garner A, Lee A, Harrison $\mathrm{K}$, et al. Comparative analysis of multiple-casualty incident triage algorithms. Ann Emerg Med 200I;38:54I-8.

I9. Risavi BL, Salen PN, Heller MB, et al. A two-hour intervention using START improves prehospital triage of mass casualty incidents. Prehosp Emerg Care 200I;5 I97-9.

20. Romig LE. Pediatric triage. A system to JumpSTART your triage of young patients at MCIs. JEMS 2002;27:52-8.

2I. United Nations Department of Peacekeeping Operations. Medical support manual for United Nations peacekeeping operations. 2nd ed. New York: Department of Peacekeeping Operations; I999. Available: www.un.org/Depts/dpko/training/tes _publications/books/medical_support/med_support.pdf (accessed 2006 Sept 25).

22. Ferreira FL, Bota DP, Bross A, et al. Serial evaluation of the SOFA score to predict outcome in critically ill patients. JAMA 200I;286:1754-8.

23. Knaus WA, Zimmerman JE, Wagner DP, et al. APACHE-acute physiology and chronic health evaluation: a physiologically based classification system. Crit Care Med I98I;9:59I-7.

24. Knaus WA, Draper EA, Wagner DP, et al. APACHE II: a severity of disease classification system. Crit Care Med I985;13:818-29.

25. Knaus WA, Wagner DP, Draper EA, et al. The APACHE III prognostic system. Risk prediction of hospital mortality for critically ill hospitalized adults. Chest I99I; Ioo: 6 19-36.

26. Marshall JC. Organ dysfunction as an outcome measure in clinical trials. Eur J Surg Suppl 1999;584:62-7.

27. Maziak DE, Lindsay TF, Marshall JC, et al. The impact of multiple organ dysfunction on mortality following ruptured abdominal aortic aneurysm repair. Ann Vasc Surg 1998;12:93-100.

28. Cabre L, Mancebo J, Solsona JF, et al. Multicenter study of the multiple organ dysfunction syndrome in intensive care units: the usefulness of Sequential Organ Failure Assessment scores in decision making. Intensive Care Med 2005;31:927-33.

29. Peres Bota D, Melot C, Lopes Ferriera F, et al. The Multiple Organ Dysfunction Score (MODS) versus the Sequential Organ Failure Assessment (SOFA) score in outcome prediction. Intensive Care Med 2002;28:16I9-24.

30. Oda S, Hirasawa H, Sugai T, et al. Comparison of Sepsis-related Organ Failure Assessment (SOFA) score and CIS (cellular injury score) for scoring of severity for patients with multiple organ dysfunction syndrome (MODS). Intensive Care Med 2000;26:1786-93.

3I. Vincent JL, Moreno R, Takala J, et al. The SOFA (Sepsis-related Organ Failure Assessment) score to describe organ dysfunction/failure. On behalf of the Working Group on Sepsis-related Problems of the European Society of Intensive Care Medicine. Intensive Care Med 1996;22:707-10.

32. Society of Critical Care Medicine Ethics Committee. Consensus statement on the triage of critically ill patients. JAMA I994;271:1200-3.

33. Task Force of the American College of Critical Care Medicine, Society of Critica Care Medicine. Guidelines for intensive care unit admission, discharge, and triage. Crit Care Med I999;27:633-8.

34. Sinuff T, Kahnamoui K, Cook DJ, et al. Rationing critical care beds: a systematic review. Crit Care Med 2004;32:1588-97.

35. Tallgren M, Klepstad P, Petersson J, et al. Ethical issues in intensive care - a survey among Scandinavian intensivists. Acta Anaesthesiol Scand 2005;49:1092-100.

36. Ryan CM, Schoenfeld DA, Thorpe WP, et al. Objective estimates of the probability of death from burn injuries. $N$ Engl J Med I998;338:362-6.

37. Kilgo PD, Meredith JW, Osler TM. Incorporating recent advances to make the TRISS approach universally available. J Trauma 2006;60:1002-8.

38. Boyd CR, Tolson MA, Copes WS. Evaluating trauma care: the TRISS method. Trauma Score and the Injury Severity Score. J Trauma I987;27:370-8.

39. Brindley PG, Markland DM, Mayers I, et al. Predictors of survival following in-hospital adult cardiopulmonary resuscitation. CMAJ 2002;167(4):343-8.

40. Fowler RA, Lapinsky SE, Hallett D, et al. Critically ill patients with severe acute respiratory syndrome. JAMA 2003;290:367-73

4I. Rubenfeld GD, Caldwell E, Peabody E, et al. Incidence and outcomes of acute lung injury. NEngl J Med 2005;353:1685-93.

42. Devlin J, O'Grady J. Indications for referral and assessment in adult liver transplantation: a clinical guideline. British Society of Gastroenterology. Gut 1999;45(Suppl 6):VII-VI22.

43. American Society for Transplant Physicians (ASTP)/American Thoracic Society(ATS)/European Respiratory Society(ERS)/International Society for Heart and Lung Transplantation(ISHLT). International guidelines for the selection of lung transplant candidates. Am J Respir Crit Care Med ig98;158:335-9.

44. UKT Cardiothoracic Advisory Group. National protocol for the assessment of cardio thoracic transplant patients. Bristol (UK): The Group; 2002. Available: www uktransplant.org.uk/ukt/about_transplants/organ_allocation/cardiothoracic/national _protocols_and_standards/protocols_and_standards/patient_assessment.jsp (accessed 2006 Sept 25).

45. Cardiac Transplantation. 24th Bethesda Conference. November 5-6, I992. J Am Coll Cardiol I993;22:I-64.

46. University of Toronto Joint Centre for Bioethics Pandemic Influenza Working Group. Stand on guard for thee: ethical considerations in preparedness planning for pandemic influenza. Toronto: University of Toronto Joint Centre for Bioethics; 2005. Available: www.utoronto.ca/jcb/home/documents/pandemic.pdf (accessed 2006 Sept 25).

47. Frykberg ER. Medical management of disasters and mass casualties from terrorist bombings: how can we cope? J Trauma 2002;53:20I-I2.

48. Hick JL, O'Laughlin DT. Concept of operations for triage of mechanical ventilation in an epidemic. Acad Emerg Med 2006;13:223-9.

Correspondence to: Dr. Michael D. Christian, Rm. $2 U$, McMaster University Medical Centre, I200 Main St. W., Hamilton ON L8N 3Z5; fax 905 523-I224; michael.christian@utoronto.ca 